\title{
INFLUENCIA DE LOS MÉTODOS DIDÁCTICOS EN EL RENDIMIENTO ACADÉMICO EN MATEMÁTICAS DE LOS ALUMNOS DE LA FACULTAD DE CIENCIAS CONTABLES Y FINANCIERAS DE LA UNIVERSIDAD NACIONAL JORGE BASADRE GROHMANN
}

\author{
Responsable : Lic. Rolando Wilman Vásquez Jaico, \\ Miembros : Mgr. CPC. Betty Cohaila Calderón \\ Eco. Juan Cáceres Mamani \\ CPC. Alexis Alpaca Cusicanqui
}

\section{RESUMEN}

En este trabajo se reflexiona sobre las caracteristicas de una enseñanza de la matemática que sea eficaz para el logro del aprendizaje significativo de los alumnos. Los métodos didácticos inciden en el rendimiento académico y juntos se convierten en una medida para el aprendizaje logrado en el aula. En este campo se hallan correlaciones sustanciales entre los métodos didácticos y el rendimiento académico en matemática en estudiantes universitarios basándose en el promedio ponderado.

Palabras clave: Educación matemática, métodos didácticos, unidad didáctica, rendimiento académico, herramientas computarizadas, problemas contextualizados.

\section{ABSTRACT}

In this work it is reflected on the characteristics of an education of the mathematical one that is effective for the profit of the significant learning of the students. The didactic methods affect the academic yield and together they become a measurement for the learning obtained in the classroom. In this field are substantial correlations between the didactic methods and mathematical the academic yield in of university students being based on the weighed average.

Key words: Mathematical education, didactic methods, didactic unit, academic yield, computerized tools.

\section{INTRODUCCIÓN}

El conocimiento matemático forma parte del acervo cultural de nuestra sociedad; es una disciplina cuya construcción empirica e inductiva surge de la necesidad y el deseo de responder y resolver situaciones provenientes de los más variados ámbitos, tanto de la matemática misma como del mundo de las ciencias naturales, sociales, del arte y la tecnología; su construcción y desarrollo es una creación del ser humano, ligada a la historia y a la cultura.

Las matemáticas constituyen la base sobre la que se construyen los modelos cientificos, toman parte en el proceso de modelización de la realidad y, en muchas ocasiones, han servido como medio de validación de estos modelos. Por ejemplo, han sido cálculos matemáticos los que permitieron, mucho antes de que pudiesen ser observados, el descubrimiento de la existencia de los últimos planetas de nuestro sistema solar. La perspectiva histórica muestra claramente que las matemáticas son un conjunto de conocimientos en evolución continua y que en dicha evolución desempeña a menudo un papel de primer orden la necesidad de resolver determinados problemas prácticos (o internos a las propias matemáticas) y su interrelación con otros conocimientos.

\section{1) Planeamiento de la Investigacion}

Las instituciones universitarias son un tipo de organizaciones que tienen problemas para adaptarse a cambios e innovaciones ya que, de entrada, son reacias a la introducción de innovaciones y poco flexibles para adaptarse a las mismas. La manera eficaz de introducir innovaciones en una institución universitaria es un tema sobre el que hay importantes desacuerdos, los cuales están relacionados con la manera de entender la relación que se establece entre las instituciones y las personas en las que se encarna dicha institución.

Los profesores de matemáticas se incorporan al mundo laboral universitario por concurso de méritos a nivel nacional, bajo una reglamentación que tiene más de 30 años y aislada de la realidad. Este concurso, que no exige como requisito una formación en pedagogia o en didáctica de la matemática, permite que los matemáticos se incorporen a la labor docente universitaria sin ninguna dificultad. En ningún momento del concurso se tuvo en cuenta la didáctica; el objetivo era demostrar al jurado que tenian un buen dominio de las matemáticas.

De esta manera, luego de terminar sus estudios de matemáticas tienen su primera experiencia como profesor. La enseñanza se hace siguiendo el modelo de sus antiguos profesores, sin cuestionarse la idoneidad de este tipo de enseñanza. Puesto que la mayor parte de su formación universitaria consistió en recibir la transmisión de contenidos formales organizados de manera deductiva, los profesores también se dedican a explicar los contenidos matemáticos de manera magistral, descontextualizada y de manera deductiva (también es la forma de presentación de los contenidos en la mayoría de los libros de texto).

Gran parte de los profesores de matemáticas de la UNJBG han descubierto que los alumnos no los entienden, y que cuando vuelven a repetir su explicación, no sólo no los entienden sino que, además, no los escuchan, y que cuando sus alumnos le preguntan "¿esto para qué sirve?" ellos no tienen claro que contestar. La dinámica de las clases les lleva al convencimiento de que tienen que cambiar su manera de enseñar las matemática, ¿pero cuál podría ser la alternativa? 
Por una parte, se constata que los alumnos no entienden, pero el profesor, a su vez, no entiende por qué los alumnos no le entienden, puesto que la explicación que los docentes hacen es clara y muy ordenada. Dicho de otra manera, los profesores no tienen una manera alternativa de enseñar la matemática diferente de la clase magistral, que evidentemente no funciona.

En la UNJBG, como en otras universidades peruanas, existen diferentes profesores que proponen una enseñanza de las matemáticas a partir de la resolución de problemas y hacen ver a los alumnos que las matemáticas se pueden aplicar a situaciones de la vida real. Se plantea, en definitiva, pasar de enseñar teorias matemáticas acabadas a enseñar a "hacer matemáticas" y de ofrecer una imagen de las matemáticas con capacidad de responder a la pregunta "esto para qué sirve". Estos intentos de renovación se van concretando en herramientas computarizadas que pretenden que el alumno descubra las aplicaciones de las matemáticas a las situaciones reales; ya que de esta manera se cree conseguir la motivación de los alumnos. Estas herramientas ponen el énfasis sobre los procesos de matematización de situaciones reales. La causa de este énfasis está en la suposición de que la principal dificultad que impide el aprendizaje de las matemáticas a muchos alumnos, es la falta de motivación causada por una presentación de la matemática exclusivamente centrada sobre ella misma, y muy alejada de las otras ciencias.

Los profesores que son conscientes de sus dificultades para enseñar matemáticas a sus alumnos, han encontrado una alternativa a la enseñanza magistral descontextualizada, han optado por la alternativa "constructivista", llegando al convencimiento de que la estructura que tenia que seguir una unidad didáctica es la siguiente:

- Problemas contextualizados introductorios.

- Desarrollo de la unidad didáctica con problemas contextualizados de aplicación intercalados.

- Problemas contextualizados de consolidación.

El diseño de este tipo de materiales obliga a los profesores a estudiar unas matemáticas diferentes a las matemáticas que habian estudiado en la universidad. Ahora necesitan saber cuáles son las aplicaciones de las matemáticas al mundo real, cuáles son los problemas que originaron los objetos matemáticos que tienen que enseñar, etc. Es decir, se tiene que volver a estudiar matemáticas, pero unas matemáticas muy diferentes a las que habían estudiado en su formación universitaria. Esta nueva mirada a las matemáticas también sirve para darse cuenta que se sabe bastante menos matemáticas de lo que se piensa. Dicho de otra manera, el profesor debe aumentar su domino de los contenidos de la matemática universitaria.

Por lo tanto, el profesor de matemática de la UNJBG tiene que desarrollar competencias profesionales:

a) Competencia en el domino de los contenidos matemáticos correspondientes al curriculo de la Facultad en la que imparte sus clases. b) Competencia en la planificación y diseño de secuencias didácticas.

c) La capacidad de gestión de las secuencias didácticas en el aula.

Los profesores deben reflexionar y analizar el contenido matemático que tendrán que enseñar, con la finalidad de aflorar sus propias creencias y actitudes hacia las matemáticas e inducir en ellos una visión constructiva y sociocultural de las mismas. Los profesores tienen que ser conscientes del papel que las matemáticas desempeñan en la ciencia, la tecnología y en la vida cotidiana y deben conocer algunos rasgos característicos de las matemáticas. En esta reflexión conviene destacar el carácter evolutivo del conocimiento matemático, el papel de la resolución de problemas y la modelización, el razonamiento, lenguaje y comunicación, la estructura lógica y naturaleza relacional de las matemáticas, así como la dialéctica entre exactitud y aproximación.

Ahora bien, se ha podido comprobar que la visión que tienen los profesores sobre la enseñanza y el aprendizaje de las matemáticas, basada fundamentalmente en su larga experiencia, está divorciada de las bases psicopedagógicas y la normativa curricular del sistema educativo. Por lo tanto, en un primer bloque, además de tener que enseñar contenidos matemáticos, la formación tiene que tender hacia una solución de dicho divorcio, además debe contemplar la satisfacción que se muestra cuando se encuentra utilidad a los contenidos de tipo pedagógico que se imparten en una clase de matemáticas y también la insatisfacción de muchos por un conocimiento de tipo psicopedagógico general que no saben cómo aplicar en la práctica.

Las consideraciones anteriores llevan a plantear que la Didáctica de las Matemáticas tiene que contemplar un segundo bloque de contenidos: el estudio de los procesos de enseñanza y aprendizaje de las matemáticas en general y de cada contenido en particular. La reflexión de tipo general sobre la enseñanza-aprendizaje de las matemáticas ha de servir para aflorar las creencias de los profesores en formación acerca de la enseñanza y el aprendizaje de dicha materia. Esta reflexión de tipo general hace indispensable un breve análisis de las nociones de competencia y comprensión matemática, esto es, sobre lo que se considera como "conocer matemáticas" desde el punto del sujeto que aprende. No parece posible tomar decisiones educativas apropiadas si no se adoptan previamente criterios claros sobre lo que se va a considerar qué es "saber matemáticas".

Un tercer bloque de contenidos debe estar dedicado al estudio del curriculo de matemáticas, al nivel de propuestas curriculares básicas y de programación de unidades didácticas. Conviene presentar una sintesis de las orientaciones curriculares para el área de matemáticas, incluyendo los fines y objetivos, contenidos y evaluación, asi como las principales caracteristicas de los principios y estándares para las matemáticas universitarias. Esta última información aportará a los profesores una visión complementaria y 
critica de las orientaciones propuestas por los órganos competentes de las universidades. Respecto del diseño y gestión de unidades didácticas hay que tener presente los principales elementos a tener en cuenta en la planificación, gestión y evaluación de las unidades.

Un cuarto bloque de contenidos debe dedicarse al estudio de los recursos didácticos utilizables en la enseñanza y aprendizaje de las matemáticas. Conviene presentar una perspectiva general de los recursos, incluyendo desde los libros de texto, materiales manipulativos, gráficos y textuales, asi como los recursos tecnológicos (calculadoras, ordenadores, internet, etc.).

El profesor debe lograr una actitud propicia al uso de materiales manipulativos de toda indole, incardinados como elementos de las situaciones didácticas, pero al mismo tiempo es necesario que construya una actitud crítica al uso indiscriminado de tales recursos. Hay que tener presente que el material manipulativo (sea tangible o gráfico-textual) puede ser un puente entre la realidad y los objetos matemáticos, pero es necesario adoptar precauciones para no caer en un empirismo ciego ni en un formalismo estéril.

\section{2) Marco de Referencia}

El fin específico de la didáctica de las matemáticas como campo de investigación es el estudio de los factores que condicionan los procesos de enseñanza y aprendizaje de las matemáticas y el desarrollo de programas de mejora de dichos procesos. Para lograr este objetivo, la didáctica de las matemáticas debe considerar las contribuciones de diversas disciplinas como la psicologia, pedagogia, filosofia, sociologia, etc. (Godino, 1991). En el desarrollo de la didáctica de la matemática, se han desarrollado nuevas herramientas e incorporado otras nociones de marcos teóricos relacionados que permiten describir de una manera detallada las interacciones que ocurren en el aula de matemáticas. Las nociones de patrón de interacción, negociación de significados, normas sociomatemáticas, aportadas por el interaccionismo simbólico son sin duda herramientas útiles para abordar esta problemática.

Asímismo, nos parece necesario tener en cuenta nociones aportadas por teorias psicológicas del aprendizaje, como la "zona de desarrollo próximo" (Vygotsky, 1979) y los supuestos del aprendizaje verbal significativo basado en la recepción (Godino, 2003).

Figura Nº1: Educación Matemática

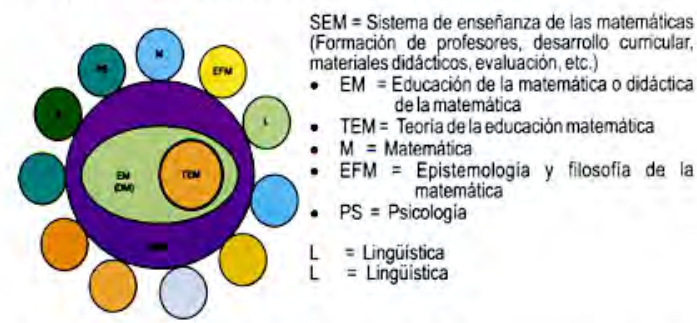

Fuente: Relaciones de la Didáctica de la Matemática con otras disciplinas y sistemas (Steiner,1990)
Steiner (1990) representa mediante el diagrama de la Figura 1 la disciplina Educación Matemática (EM) que está relacionada, formando parte de él, con otro sistema complejo social que llamaremos Sistema de Enseñanza de la Matemática (SEM) - denominado por Steiner "Educación Matemática y Enseñanza"-, representado en el diagrama por el circulo de trazo más grueso exterior a la EM. La actividad de teorización (TEM) es vista por Steiner como un componente de la Educación Matemática, $\mathrm{y}$, por ende, del sistema más amplio que hemos denominado SEM, que constituye el sistema de enseñanza de las matemáticas.

\section{Figura $\mathrm{N}^{\circ}$ 02: \\ Modelo \\ tetraédrico de \\ Higginson para \\ la Educación \\ Matemática}

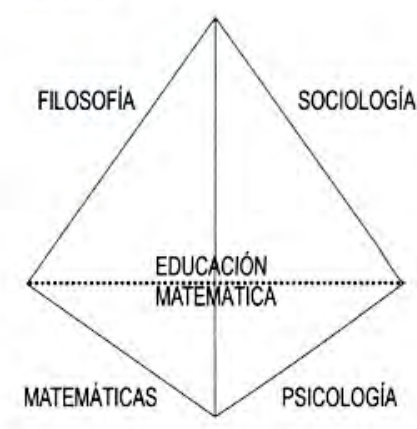

Un modelo de las relaciones de la Educación Matemática con otras disciplinas es propuesto por Higginson (1980), quien considera a la matemática, psicología, sociología y filosofía como las cuatro disciplinas fundacionales de esa educación. Visualiza la Educación Matemática en términos de las interacciones entre los distintos elementos del tetraedro cuyas caras son dichas cuatro disciplinas, Figura 2.

Estas distintas dimensiones de la Educación Matemática asumen las preguntas básicas que se plantean en nuestro campo:

- ¿Qué enseñar? (Matemáticas)

- ¿Porqué? (Filosofia)

- ¿Aquién y dónde? (Sociología)

- ¿Cuándo y cómo? (Psicologia)

Por último, los ciudadanos con competencia matemática tienden a apreciar las matemáticas como una disciplina dinámica, cambiante e importante que, a menudo, les resulta útil para sus necesidades

Para describir más claramente el área de conocimiento de matemáticas deben distinguirse tres elementos,Figura 3:

- Las situaciones o contextos en que se sitúan los problemas;

- El contenido matemático del que hay que valerse para resolver los problemas, organizado según ciertas ideas principales;

- Las competencias que deben activarse para vincular el mundo real en el que se generan los problemas con las matemáticas, y, por tanto, para resolver los problemas. 
Figura $\mathrm{N}^{0}$ 03: Los elementos del área de conocimiento 0 matemáticas.

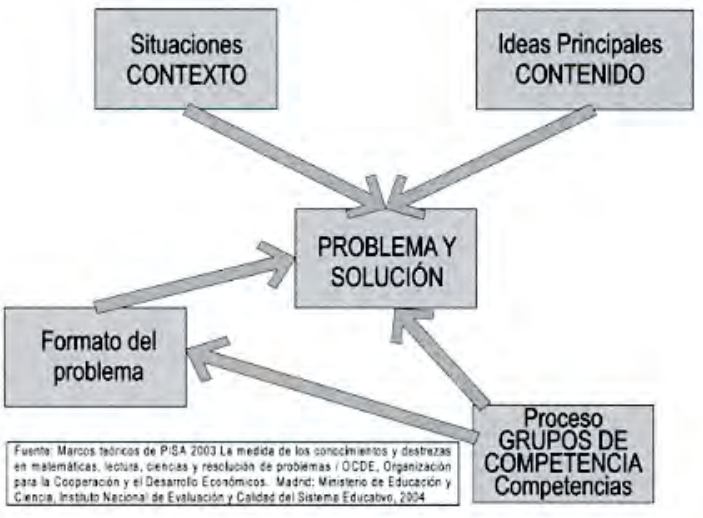

El grado de competencia matemática de una persona se observa en el modo en que utiliza sus destrezas y conocimientos matemáticos al resolver problemas. Los problemas (y su resolución) pueden presentarse en una gran variedad de situaciones o contextos en la experiencia de una persona. Todo contenido que se aprende es también susceptible de ser enseñado, y se considera tan necesario planificar la intervención con respecto a los contenidos de tipo conceptual como planificarla con relación a los otros tipos de contenido.

- Los conceptos, hechos y principios.

- Los procedimientos.

- Los valores, normas y actitudes.

En la actividad matemática, se encuentran los siguientes seis tipos de objetos:

Problemas y situaciones (cuestiones, ejercicios, etc.) Lenguaje (términos, expresiones, gráficos, etc.)

Acciones (, técnicas, algoritmos, etc.)

Conceptos (definiciones o reglas de uso)

Propiedades de los conceptos y acciones

Argumentaciones (inductivas, deductivas, etc.)

Estos objetos están relacionados unos con otros. El lenguaje es imprescindible para describir los problemas, acciones, conceptos, propiedades y argumentaciones. Los conceptos y propiedades deben ser recordados al realizar las tareas, las argumentaciones sirven para justificar las propiedades. En la actividad matemática aparecen también una serie de procesos que se articulan en su estudio, cuando los estudiantes interaccionan con las situaciones - problemas, bajo la dirección y apoyo del profesor. Los Principios y Estándares 2000 del NCTM resaltan la importancia de los procesos matemáticos, en la forma que resumimos a continuación.

1. Resolución de problemas.

2. Representación.

3. Comunicación.

4. Justificación.

5. Conexión.

6. Institucionalización.

Estos procesos se deben articular a lo largo de la enseñanza de los contenidos matemáticos organizando tipos de situaciones didácticas que los tengan en cuenta.
Acontinuación los describimos brevemente.

\section{3) Elementos Básicos del Proyecto}

\section{En consecuencia, se formula el Problema general} de investigación:

¿Cuál es la influencia de los métodos didácticos de matemática en el rendimiento académico de los alumnos en la Facultad de Ciencias Contables y Financieras de la Universidad Nacional Jorge Basadre Grohmann?

\section{Las razones por las que se emprendió el proyecto son:}

- Nivel teórico: Esta investigación sirve para conocer la relación existente, de acuerdo con un modelo lineal, entre el rendimiento académico y los métodos didácticos utilizados en la enseñanza de la matemática; además, puede ser una base para futuras investigaciones vinculadas al tema.

- Nivel práctico: Este trabajo sirve para seguir precisando los factores que interfieren en el adecuado desempeño académico de los docentes y estudiantes; además de alcanzar información que ayude a desarrollar la didáctica de los profesores de matemáticas dirigida a optimizar su rendimiento académico. El proyecto prueba la hipótesis general siguiente: Los métodos didácticos en matemática influyen en el rendimiento académico en los alumnos de la Facultad de Ciencias Contables y Financieras de la Universidad Nacional Jorge Basadre Grohmann.

$\mathrm{H}_{1}$ :Existe relación entre los métodos didácticos en matemática y el rendimiento académico en los alumnos de la Facultad de Ciencias Contables y Financieras de la Universidad Nacional Jorge Basadre Grohmann.

$\mathrm{H}_{0}$ : No existe relación entre los métodos didácticos en matemática y el rendimiento académico de los alumnos de la Facultad de Ciencias Contables y Financieras en la Universidad Nacional Jorge Basadre Grohmann.

\section{El Objetivo General:}

Establecer la influencia de los métodos didácticos de matemática en el rendimiento académico de los alumnos en la Facultad de Ciencias Contables y Financieras de la Universidad Nacional Jorge Basadre Grohmann.

\section{MATERIAL Y MÉTODOS}

\section{1) Método}

\section{Tipo de Investigación}

Debido al problema de la investigación y a las caracteristicas de la muestra, el estudio es de tipo correlacional y tiene como propósito medir el grado de relación que existe entre dos o más conceptos 0 variables, y esto se ajusta a la definición brindada por 
Hernández, Fernández y Baptista (1991), acerca de los estudios correlacionales.

\section{Diseño de Investigación}

El diseño de la investigación es no experimental, no existe manipulación activa de alguna variable. Además, se trata de un diseño transeccional transversal, porque se establece la relación de variables medidas en una muestra en un único momento del tiempo (Hernández, Fernández, y Baptista; 1991).

\section{2) Población y Muestra}

La población está conformada por estudiantes universitarios, de ambos sexos, del primera año de la Facultad de Ciencias Contables y Financieras de la Universidad Nacional Jorge Basadre Grohmann. La muestra estuvo constituida por 91 estudiantes de ambos sexos, que cursaron estudios en el primer año de la Facultad de Ciencias Contables y Financieras de la UNJBG, el año 2005. Muestra que constituye aproximadamente el $76,47 \%$ de la población del primer año. Las características de esta muestra son las siguientes:

Tabla N ${ }^{\circ} 01$ : Caracteristicas de la Muestra

\begin{tabular}{|c|c|c|c|c|}
\hline \multirow{2}{*}{$\begin{array}{c}\text { Número } \\
\text { Total }\end{array}$} & \multicolumn{2}{|c|}{$\begin{array}{c}\text { Sexo de la Muestra } \\
\text { Femenino }\end{array}$} & \multicolumn{2}{c|}{ Masculino } \\
\cline { 2 - 5 } & N & $\%$ & N & $\%$ \\
\cline { 2 - 5 } & 42 & $46,15 \%$ & 49 & $53,85 \%$ \\
\hline 91 & & & & \\
\hline
\end{tabular}

En esta muestra sólo fueron considerados estudiantes que, según los registros, cuentan con todas las evaluaciones en el curso de matemática del primer año de Contabilidad.

\section{3) Variables}

\section{Variables Relacionadas:}

Variable Independiente: Métodos didácticos en matemáticas. Sistema complejo y heterogéneo que incluye teoria, desarrollo y práctica relativa a la enseñanza y aprendizaje de las matemáticas. Su finalidad es identificar, caracterizar, y comprender los fenómenos y procesos que condicionan la enseñanza y el aprendizaje de las matemáticas

Variable Dependiente: Rendimiento académico. Variable de tipo cuantitativo. Categorizada como rendimiento alto, medio, bajo y deficiente, según la tabla elaborada por Reyes Murillo (1988). El indicador para esta variable es el promedio ponderado de cada estudiante, luego de culminar el año académico. Representa el nivel de eficacia en la consecución de los objetivos curriculares para las diversas asignaturas, y se expresa mediante un calificativo o promedio ponderado basado en el sistema vigesimal; es decir. las notas variarán de 0 a 20 puntos, donde el puntaje de 10 ó menos es reprobatorio.

Variables Controladas: Examen. Es una situación 0 acto evaluativo donde se miden ciertas aptitudes, nivel de suficiencia, de conocimientos, habilidades o rasgos psicológicos del sujeto examinado. El grado de eficiencia en la ejecución de la prueba determinará la aprobación o reprobación del examinado, cuyo calificativo puede ser cuantitativo o cualitativo. Año de Estudio (estudiantes universitarios del primer año).Facultad donde estudian los sujetos examinados (Facultad de Ciencias Contables y Financieras), indica un nivel de confianza del $95 \%$, tanto para las correlaciones simples como para las diferencias y regresiones múltiples encontradas.

\section{4) Técnicas de Recolección de Datos}

Para la recolección de datos se utilizó la evaluación psicométrica de las variables psicológicas estudiadas, como son: la ansiedad ante los exámenes, los rasgos de personalidad, el auto concepto y la asertividad, aplicada a los estudiantes que conforman la muestra en dos jornadas de variada duración. Luego, se procedió a la calificación de las pruebas con los baremos más actualizados y adecuados para la muestra a que se tuvo acceso, y se realizó el análisis estadistico de los resultados a través del paquete de análisis estadistico para la investigación en ciencias sociales SPSS (Versión $12,0)$, con el fin de comprobar la hipótesis planteada. Para acceder a los datos, de la variable rendimiento académico, se utilizó la técnica documental, al acceder a los promedios ponderados de los estudiantes de la muestra, luego de culminar su primer año de Estudios Superiores.

\section{5) Análisis Estadístico}

Para el análisis estadistico se emplearon los siguientes estadigrafos y pruebas estadisticas: media aritmética, desviación estándar, varianza, coeficiente de correlación lineal de Pearson, ecuación de análisis de regresión múltiple.

Nivel de Significación. Al 0,05, que indica un nivel de confianza del $95 \%$, tanto para las correlaciones simples como para las diferencias y regresiones múltiples encontradas.

\section{ANÁLISIS DELOS RESULTADOS}

Luego de la obtención del nivel de rendimiento académico se procedió a analizar la información, cuyos resultados presentamos a continuación.

\section{1) Definiciones acerca del Rendimiento Académico}

Todo proceso educativo busca permanentemente mejorar el aprovechamiento del alumno. En este sentido, la variable dependiente clásica en la educación universitaria es el rendimiento 0 aprovechamiento académico. El rendimiento en sí y el rendimiento académico son definidos por la Enciclopedia de Pedagogia / Psicologia de la siguiente manera: "Del latin reddere (restituir, pagar) el rendimiento es una relación entre lo obtenido y el esfuerzo empleado para obtenerlo". Es un nivel de éxito en la universidad, en el trabajo, etc. A 
al hablar de rendimiento en la universidad, nos referimos al aspecto dinámico de la institución universitaria. El problema del rendimiento académico se resolverá de forma científica cuando se encuentre la relación existente entre el trabajo realizado por el profesor y los alumnos, de un lado, y la educación (es decir, la perfección intelectual y moral lograda por éstos) de otro. Al estudiar científicamente el rendimiento, es básica la consideración de los factores que intervienen en él. Por lo menos en lo que a la instrucción se refiere, existe una teoria que considera que el rendimiento académico se debe predominantemente a la inteligencia; sin embargo, lo cierto es que ni si quiera en el aspecto intelectual del rendimiento la inteligencia es el único factor. Al analizarse el rendimiento escolar, deben valorarse los factores ambientales como la familia, la sociedad y el ambiente escolar.

Además, el rendimiento académico es entendido por Pizarro (1985) como una medida de las capacidades respondientes 0 indicativas que manifiestan, en forma estimativa, lo que una persona ha aprendido como consecuencia de un proceso de instrucción o formación. El mismo autor, ahora desde una perspectiva propia del alumno, define el rendimiento como una capacidad respondiente de éste frente a estímulos educativos, susceptible de ser interpretado según objetivos o propósitos educativos preestablecidos. Este tipo de rendimiento académico puede ser entendido en relación con un grupo social que fija los niveles mínimos de aprobación ante un determinado cúmulo de conocimientos o aptitudes (Carrasco, 1985).

Por su lado, Kaczynska (1986) afirma que el rendimiento académico es el fin de todos los esfuerzos y todas las iniciativas escolares del maestro, de los padres, de los mismos alumnos; el valor de la institución educativa y el maestro se juzgan por los conocimientos adquiridos por los alumnos.

En tanto que Nováez (1986) sostiene que el rendimiento académico es el quantum obtenido por el individuo en determinada actividad académica. El concepto de rendimiento está ligado al de aptitud, y sería el resultado de ésta, de factores volitivos, afectivos y emocionales, además de la ejercitación.

Chadwick (1979) define el rendimiento académico como la expresión de capacidades y de características psicológicas del estudiante desarrolladas y actualizadas a través del proceso de enseñanza-aprendizaje que le posibilita obtener un nivel de funcionamiento y logros académicos a lo largo de un periodo o semestre, que se sintetiza en un calificativo final (cuantitativo en la mayoria de los casos) evaluador del nivel alcanzado.

El rendimiento académico es un indicador del nivel de aprendizaje alcanzado por el alumno. Por ello, el sistema educativo brinda tanta importancia a dicho indicador. En tal sentido, el rendimiento académico se convierte en una "tabla imaginaria de medida" para el aprendizaje logrado en el aula, que constituye el objetivo central de la educación. Sin embargo, en el rendimiento académico intervienen muchas otras variables externas al sujeto, como la calidad del maestro, el ambiente de clase, la familia, el programa educativo, etc., y variables psicológicas o internas, como la actitud hacia la asignatura, la inteligencia, la personalidad, el auto concepto del alumno, la motivación, etc. Es pertinente dejar establecido que aprovechamiento escolar no es sinónimo de rendimiento académico. El rendimiento académico o escolar parte del presupuesto de que el alumno es responsable de su rendimiento. En tanto que el aprovechamiento escolar está referido, más bien, al resultado del proceso enseñanza-aprendizaje, de cuyos niveles de eficiencia son responsables tanto el que enseña como el que aprende.

\section{2) Características del Rendimiento Académico}

Garcia y Palacios (1991), después de realizar un análisis comparativo de diversas definiciones del rendimiento escolar, concluyen que hay un doble punto de vista, estático y dinámico, que atañen al sujeto de la educación como ser social. En general, el rendimiento escolar es caracterizado del siguiente modo:

- En su aspecto dinámico responde al proceso de aprendizaje, como tal está ligado a la capacidad y esfuerzo del alumno.

- En su aspecto estático comprende al producto del aprendizaje generado por el alumno y expresa una conducta de desaprovechamiento.

- Está ligado a medidas de calidad y a juicios de valoración.

- El rendimiento es un medio y no un fin en sí mismo.

- El rendimiento está relacionado a propósitos de carácter ético que incluye expectativas económicas, lo cual hace necesario un tipo de rendimiento en función del modelo social vigente.

\section{3) El Rendimiento Académico en el Perú}

En consonancia con esa caracterización y en directa relación con los propósitos de la investigación, es necesario conceptuar el rendimiento académico. Para ello se requiere previamente considerar dos aspectos básicos del rendimiento: el proceso de aprendizaje y la evaluación de dicho aprendizaje. El proceso de aprendizaje no será abordado en este trabajo. Sobre la evaluación académica hay una variedad de postulados que pueden agruparse en dos categorias: aquellos dirigidos a la consecución de un valor numérico (u otro) y aquellos encaminados a propiciar la comprensión en términos de utilizar también la evaluación como parte del aprendizaje. En el presente trabajo interesa la primera categoria, que se expresa en las calificaciones. Las calificaciones son las notas o expresiones cuantitativas o cualitativas con las que se valora o mide el nivel del rendimiento académico en los alumnos. Las calificaciones son el resultado de los exámenes o de la evaluación continua a que se ven sometidos los estudiantes. Medir o evaluar los rendimientos escolares es una tarea compleja que exige del docente obrar con la máxima objetividad y precisión (Fernández Huerta, 1983; cit. porAliaga, 1998b).

En el sistema educativo peruano, en especial en las universidades y en este caso específico en la UNJBG, las calificaciones se basan en el sistema vigesimal, es decir, de 0 a 20 (Miljanovich, 2000). Sistema en el cual el puntaje obtenido se traduce a la categorización del logro de aprendizaje, el cual puede variar desde aprendizaje bien logrado hasta aprendizaje deficiente, basándonos en el siguiente cuadro (DIGEBARE, 1980; cit. por Reyes Murillo, 1988): 
Tabla No 02: Categorización del Nivel de Rendimiento Académico (según la DIGEBARE del Ministerio de Educación)

\begin{tabular}{|l|l|}
\hline Notas & Valorización \\
\hline $15-20$ & Aprendizaje bien logrado \\
\hline $11-14$ & Aprendizaje regularmente logrado \\
\hline $10-0$ & Aprendizaje deficiente \\
\hline
\end{tabular}

Fuente: Ministerio de Educación. Dirección General de Educación Básica y Regular (DIGEBARE): Guia de Evaluación del Educando . Lima, 1980.

Reyes Murillo (1988) elaboró una tabla diferente para la valoración del aprendizaje según las calificaciones obtenidas que se muestran en la siguiente tabla:

Tabla $\mathbf{N}^{\circ}$ 03: Categorización del Nivel de Rendimiento Académico (según Edith Reyes Murillo)

\begin{tabular}{|c|l|}
\hline Notas & Valorización del Aprendizaje Logrado \\
\hline $20-15$ & Alto \\
\hline $14,99-13$ & Medio \\
\hline $12,99-11$ & Bajo \\
\hline $10,99-$ menos & Deficiente \\
\hline
\end{tabular}

Fuente: Reyes Murillo, Edith T. Influencia del programa curricular y del trabajo docente en el aprovechamiento escolar en Historia del Perú de alumnos del 3er Grado de Educación Secundaria. Lima, 1988.

Aqui se observa un mayor nivel de exigencia para la valoración del aprendizaje logrado, al catalogar un aprendizaje bien logrado en un intervalo más breve dentro de las calificaciones obtenidas, lo cual permite una mayor seguridad de que el objetivo central de la educación, el aprendizaje del alumno, se haya alcanzado

\section{4) El Rendimiento Académico y su Relación con algunas Variables Psicológicas}

El rendimiento académico en general se ve unido a muchas variables psicológicas, una de ellas es la inteligencia, que se le relaciona de modo moderado a alto, en diversas poblaciones estudiantiles. Un panorama algo diferente presentan las correlaciones con las variables denominadas "comportamientos afectivos relacionados con el aprendizaje". Las correlaciones de la actitud general hacia la institución educativa, si bien son significativas, son menores que las correlaciones de la actitud hacia una asignatura determinada. En cuanto al rendimiento en algunas asignaturas, como por ejemplo la matemática, Bloom (1977, cit. por Reyes Tejada, 2003) comunica resultados de estudios univariados en los cuales se hallan correlaciones sustanciales entre la inteligencia y el aprovechamiento en aritmética en estudiantes secundarios estadounidenses.

Comenzaremos identificando el nivel de rendimiento académico de los estudiantes del primer año de Contabilidad, basándose en el promedio ponderado obtenido por la muestra al concluir el año académico, que son presentados en la Tabla No.04. Estos promedios han sido categorizados según la tabla de valoración del rendimiento académico, elaborada por Reyes Murillo
(1988) que se muestran en la Tabla No. 02 y que categoriza a esta variable en los niveles: alto (calificaciones de 15 a 20 puntos), medio (de 13 hasta $14,99)$, bajo (11 a 12,99) y deficiente $(10,99$ a menos) rendimiento académico, lo que implica para el sistema educativo peruano el nivel de aprendizaje logrado por el alumno (Aliaga,1998).

Tabla $\mathbf{N}^{\circ}$ 04: Nivel de Rendimiento Académico de los Estudiantes.

\begin{tabular}{|c|c|c|c|}
\hline $\begin{array}{c}\text { Nivel de } \\
\text { Rendimiento }\end{array}$ & Frecuencia & $\begin{array}{c}\text { Porcentaje } \\
\text { Válido }\end{array}$ & $\begin{array}{c}\text { Porcentaje } \\
\text { Acumulado }\end{array}$ \\
\hline Alto & 13 & $14,29 \%$ & $14,29 \%$ \\
\hline Medio & 17 & $18,68 \%$ & $32,97 \%$ \\
\hline Bajo & 38 & $41,76 \%$ & $74,73 \%$ \\
\hline Deficiente & 23 & $25,27 \%$ & $100,00 \%$ \\
\hline Total & 91 & $100,00 \%$ & \\
\hline
\end{tabular}

La Tabla No. 04 nos indica que el $41,76 \%$ de los estudiantes que conforman la muestra se ubica en el nivel bajo, en lo que respecta a su rendimiento académico; seguido por un $25,27 \%$ que se encuentran en un nivel deficiente, y que sumado estos dos valores, representan a un $85,71 \%$ (más que la mitad de la muestra) cuyo rendimiento académico está por debajo de lo esperado; observándose sólo a un $14,29 \%$ con un alto nivel de rendimiento académico, lo que en términos educativos implica un aprendizaje mal logrado. Estos son confirmados desde otro punto de vista por los estadisticos descriptivos correspondientes a esta variable, en donde la media aritmética es 11,51, que de acuerdo con la tabla de categorización del rendimiento académico de Reyes Murillo (1988), corresponde a un nivel bajo, que implica un aprendizaje minimamente logrado.

\section{DISCUSIÓNDELOS RESULTADOS}

En los resultados se observa, en primer lugar, que el nivel de rendimiento académico de los estudiantes evaluados se encuentra en su mayoria en el nivel bajo $(41,76 \%)$ que significa un aprendizaje mínimamente logrado; asi como un $25,27 \%$ en total correspondiente a los niveles bajo y deficiente, que representan un nivel no satisfactorio del aprendizaje alcanzado por los estudiantes en su primer año de estudios. Esto implica que sólo el $14,29 \%$ de la muestra alcanzó un nivel alto de rendimiento académico, lo que quiere decir un aprendizaje satisfactorio de los contenidos estudiados en el primer año de formación profesional, dejando por debajo al $85,31 \%$ con un aprendizaje medio, bajo o deficiente.

En consecuencia, se debe realizar un proceso de recogida y análisis de información que permita conocer hasta qué punto se está produciendo un buen proceso de enseñanza y aprendizaje, y qué problemas se están planteando en este proceso. La información resultante proporciona al profesor elementos para analizar criticamente su intervención educativa, detectar necesidades y tomar decisiones al respecto.

\section{CONCLUSIONES}

Las conclusiones a que se arribó al finalizar este trabajo son las siguientes: 
1. En términos generales, el rendimiento académico del grupo de estudiantes del primer año de la Facultad de Ciencias Contables y Financieras que fueron examinados en el presente trabajo, se ubica en el nivel bajo o deficiente, correspondiente a un aprendizaje minimamente logrado.

2. El número de horas que se asignen a la enseñanza de las matemáticas, en las diferentes carreras universitarias, ha de ser un número suficiente para permitir una enseñanza en la que prime la conexión entre conceptos matemáticos, la resolución de problemas, el razonamiento y la comunicación.

3. El tratamiento de la diversidad que exige la enseñanza universitaria es muy difícil y necesita de profesores preparados para afrontar la gran complejidad de las aulas universitarias.

4. El tratamiento de la diversidad requiere de profesores con un alto nivel de formación que trabajen en equipos bien coordinados.

5. No existe una adecuada transición entre la educación secundaria y la enseñanza universitaria. Dicho de otra manera, los alumnos no acceden desde la primaria con una formación adecuada en matemáticas ni finalizan la enseñanza secundaria con una formación en matemáticas adecuada para acceder a la universidad.

\section{RECOMENDACIONES}

De acuerdo con los resultados y las conclusiones se sugiere lo siguiente:

Es necesario desarrollar programas de asesoria académica dirigida a reforzar el aprendizaje de la población del primer año y programas de capacitación docente en el área de matemáticas, teniendo en cuenta que es la base para muchos de los cursos en la carrera profesional de Contabilidad.

Continuar desarrollando investigaciones dirigidas a conocer las diferentes variables que puedan estar relacionadas con el rendimiento académico de los alumnos y los métodos didácticos de la matemática.

La enseñanza de las matemáticas debe ser contextualizada, relacionada directamente con situaciones de la vida real y con gran énfasis en los algoritmos.

Trabajar de forma habitual, el razonamiento matemático, la comunicación, la modelización, la formulación y resolución de problemas.
Remplazar el papel excesivo de los libros de texto por un uso de materiales didácticos en la enseñanza de las Matemáticas y de las TIC.

\section{BIBLIOGRAFÍA}

Aliaga Tovar, J. (1998). La ubicación espontánea del asiento como función de la inteligencia, la personalidad, el rendimiento académico y el sexo. Tesis para optar el Grado Académico de Magíster en Psicologia. Mención Psicologia Educativa. Universidad Nacional Mayor de San Marcos, Lima, Perú.

Bloom, B. (1977). Caracteristicas humanas y aprendizaje escolar. Colombia

Carrasco, J. (1985). La recuperación educativa. . Editorial Anaya.España

Chadwick, C. (1979). Teorias del aprendizaje. Ed. Tecla.Santiago

Garcia, O., Palacios, R. (1991). Factores condicionantes del aprendizaje en lógica matemática. Tesis para optar el Grado de Magister. Universidad San Martin de Porres, Lima, Perú.

Godino, J. ; Batanero, C.; Font, V. (2003). Fundamentos de la enseñanza y el aprendizaje de las matemáticas para maestros. Departamento de Didáctica de la Matemática. Universidad de Granada: Granada. Distribución en Internet: http://www.ugr.es/local/jgodino/edumat-maestros/.

Kaczynska, M. (1986). El rendimiento escolar y la inteligencia. Paidós. Buenos Aires

Miljanovich Costilla, M. (2000). Relaciones entre la inteligencia general, el rendimiento académico y la comprensión de lectura en el campo educativo. Tesis para optar el Grado de Doctor en Educación. Universidad Nacional Mayor de San Marcos, Lima, Perú.

Pizarro, R. (1985). Rasgos y actitudes del profesor efectivo. Tesis para optar el Grado de Magister en Ciencias de la Educación. Pontificia Universidad Católica de Chile, Chile.

Reyes Murillo, E. (1988). Influencia del programa curricular y del trabajo docente en el aprovechamiento escolar en historia del Perú de alumnos del 3.er grado de educación secundaria. Tesis para optar el Grado Académico de Doctora en Educación. Universidad Nacional Mayor de San Marcos, Lima, Perú.

Reyes, Y. (2003). Relación entre el rendimiento académico, la ansiedad ante los exámenes, los rasgos de personalidad, el auto concepto y la asertividad en estudiantes del primer año de sicología de la UNMSM. Tesis Digitales, Lima-Perú. 